STCIDOC

Infer, Interpret \& Inspire Science
International Journal of Reproduction, Fertility \& Sexual Health (IJRFSH)

ISSN: 2377-1887

\title{
Sexual Health Issues in Women Treated for Carcinoma Breast and Carcinoma Cervix
}

\author{
Review Article
}

Sabhyata Gupta ${ }^{1 *}$, Buchun Mishra ${ }^{2}$, Dweep Jindal ${ }^{3}$

${ }^{1}$ Chairperson, Department of Gynaecology, Gynaecology Oncology and Robotic surgery, Medanta, The Medicity, Gurugram India.

${ }^{2}$ Consultant, Department of Gynaecology, Gynaecology Oncology and Robotic surgery, Medanta, The Medicity, Gurugram India.

${ }^{3}$ Associate Consultant, Department of Gynaecology, Gynaecology Oncology and Robotic surgery, Medanta, The Medicity, Gurugram India.

\section{Abstract}

The most frequently treated cancer in women, include breast and genital malignancy; of which cervical and uterine cancer are commonest. Treatment of these cancer can cause adverse short term and long term effects on sexual health.

Sexual dysfunction is common in cancer survivors. Despite marked advances in the management of these malignancies, side effects of various treatment modalities utilized; surgery, radiation, chemotherapy and hormonal treatment can lead to sexual health issues in women. Although sexual dysfunction leads to immense distress, majority of the women who have undergone treatment for breast and cervical carcinoma are not evaluated or treated appropriately for sexual dysfunction.Sexual problems and menopause related symptoms can have a significant negative impact on quality of life. Research has shown that at least 50 percent of the women treated for cervical carcinoma develop sexual dysfunction after completion of treatment.

In this article we provide an overview of the various treatment modalities and associated sexual health issues afflicting women that compromise their quality of life.

\section{Introduction}

Gynecological cancers contribute significantly to the burden of malignancies among women, with approximately one million cases worldwide. In addition, it has been noted that the incidence of breast cancer has increased globally [1]. Breast carcinoma is the most frequently encountered malignancy in women. With the improvement in management strategies, increased number of women survive breast carcinoma. This leads to breast carcinoma survivors being the largest proportion of cancer survivors. In the United States, breast cancer survivors account for 41 percent of the cancer survivors [2].

One of the important issues faced by these cancer survivors are those of sexual dysfunction. In a study conducted by Kowalczyk, patient who underwent breast surgery were reported to have a negative impact on sexual function. Presence of a sexual partner, availability of a support system and anxiety are found to be predictors of sexual function in breast cancer survivors [3]. Raggio et al., assessed breast cancer survivors at a median of 7 years after detection with breast carcinoma. In this study, the survivors were evaluated for sexual dysfunction using female sexual function index (FSFI) and the female sexual distress scale-revised (FSDS-R). They found that complaints of sexual dysfunction were reported among 77 percent of the survivors based on the Female Sexual Function Index (FSFI) [4].

Attempts to improve sexual function in breast cancer survivors have been reported. Hummel et al evaluated the use of Internet based cognitive behavioral therapy on sexual function and relationship intimacy. It was concluded that Internet based cognitive behavioral therapy has salutary effects on sexual functioning, body image and menopausal symptoms in breast cancer survivors [5].

Among the gynecological cancers, commonest are cervical and uterine cancers. Cervical carcinoma is predominantly seen in low and middle income countries. 85 percent of the cases occur in the developing countries. Among these cases, seventy percent are de-

\section{*Corresponding Author:}

Sabhyata Gupta MD (Obstetrics \& Gynaecology), FICOG,

Chairperson, Department of Gynaecology, Gynaecology Oncology and Robotic surgery, Medanta, The Medicity, Gurugram India.

E-mail: drsabhyata@yahoo.com

Received: April 27, 2020

Accepted: June 25, 2020

Published: June 30, 2020

Citation: Sabhyata Gupta, Buchun Mishra, Dweep Jindal. Sexual Health Issues in Women Treated for Carcinoma Breast and Carcinoma Cervix. Int J Reprod Fertil Sex Health. 2020;6(2):136-141. doi: http://dx.doi.org/10.19070/2377-1887-2000025

Copyright: Sabhyata Gupta 2020 . This is an open-access article distributed under the terms of the Creative Commons Attribution License, which permits unrestricted use, distribution and reproduction in any medium, provided the original author and source are credited. 
tected in Stage III or higher due to lack of awareness and proper screening. This leads to increased mortality rates. However with advances in treatment, mortality rates in breast and cervical cancer have decreased, leading to increased survival and increase in morbidity $[6,7]$. It is paramount that healthcare providers discuss sexual health issues after cancer treatment.

\section{Breast Carcinoma: Sexual Issues}

Breast cancer is treated by a combination of surgery, chemotherapy, radiation and hormonal treatment. All these modalities have various impacts on sexual health issues among women. The importance of sexual health in cancer survivors was demonstrated by a survey conducted by Livestrong in 2010 . Out of more than three thousand patients included, $24 \%$ were breast cancer survivors. Post treatment sexual function and satisfaction were ranked third among the most frequently reported concerns [9]. Sexual concerns result in significant emotional distress including sadness, depression, issues related to personal appearances, stigma and negatively impacts on personal relationship.

Surgical management of breast cancer entails surgery of either part or the entire breast and axilla, which may be either unilateral or bilateral. On assessing the impact of surgery and the type of surgery performed for breast carcinoma, it was noted that women undergoing mastectomy reported greater disruption in their lives, lower scores in term of body image. When mastectomy was compared to conservative surgery, it was reported that women undergoing mastectomy had significantly greater problems associated with sexual health. There was improvement in certain parameters over time but sexual function remained compromised [10]. The role of breast reconstructive surgery in maintaining sexual health is a subject of discussion. Although some studies suggest performing reconstructive surgery results in improved sexual function, other studies offer less promising results [11-13].

Chemotherapy is utilised in treatment of breast carcinoma in a large number of patients. In women who have not attained menopause, chemotherapy can lead to ovarian failure and early onset of menopause. This leads to hypoestrogenism causing loss of libido and other sexual dysfunction. Vulvo-vaginal atrophy is one of the commonest side effects of hypoestrogenism. Chemotherapy inherently can lead to a negative impact on global physical function, decreased interest, arousal and desire [14]. Radiotherapy is utilised in the treatment of breast carcinoma based on the treatment protocols and the pathological features of the tumour. Radiation results in locoregional adverse effects like breast pain, upper limb discomfort and lymphedema. All these are associated with a negative impact on sexual function. However exact impact of radiation alone on sexual health is difficult to determine as it is always associated with combination of surgery and chemotherapy as treatment modality [15-18].

Endocrine therapy is another important modality for treatment of breast cancer. Tamoxifen is utilized in pre menopausal women. For post menopausal women, aromatase inhibitors are prescribed as endocrine therapy to reduce cancer recurrence. Hormonal therapy may adversely impact sexual health. It was noted that 30-40 percent of women treated with tamoxifen and almost half of the patients treated with aromatase inhibitors reported sexual health issues. Increased dyspareunia and vaginal dryness are noted with the use of aromatase inhibitors as compared to tamoxifen. Furthermore, use of aromatase inhibitors is associated with increased risk of lichen sclerosus leading to severe itching, thinning of the perineal skin which causes bruising and tearing of the skin $[19,20]$.

Sexual health is often an ill addressed medical issue. Despite proven sexual health issues, less than half the women who are treated for breast cancer receive any medical attention for the same. This could be due to a combination of multiple factors such as reluctance of women to discuss and seek treatment for the sexual problems along with the possible lack of background and knowledge of sexual health issues among the primary treating physician. Halley et al., put forth possible barriers for appropriate management of sexual dysfunction.

- Sexual function was considered in terms of a physical domain and not a global issue.

- Patients were not sure as to where to seek appropriate treatment. - Lack of services [21].

\section{Cervical Cancer}

Compared to any malignancy, issues of long-term psychological, social adaptation and quality of life are highest with cervical cancer survivors. It is associated with high proportion of long term survivors and involves a gender specific organ affecting sexuality and sexual functioning. This constitutes an important dimension of quality of life. According to the SEER data there has been a decline in the incidence of cervical carcinoma in developed countries like USA. Majority of the new cases and deaths related to cervical carcinoma (approximately $85 \%$ and $90 \%$, respectively) occur in low-resource countries [22-23]. Cervical carcinoma is usually detected at an age where women are at the peak of their sexual activity and family building [24]. The most common sexual health issues noted in cervical carcinoma survivors include decreased elasticity and atrophy of vagina, decreased lubrication, loss of vaginal sensation, reduced arousal and sexual desire, dyspareunia and vaginal bleeding [25-27].

Surgical management of cervical cancer includes conization, simple hysterectomy and radical hysterectomy with lymphadenectomy based on the tumour characteristics and clinical stage. Radical hysterectomy results in a negative impact on sexual health which adversely impacts quality of life. Short term complications include shortening of vagina, dyspareunia, orgasmic problems, genital numbness and sexual dissatisfaction. Long term effects include reduction in sexual desire, lymphedema, decreased lubrication and persistent numbness [28-31]. Nerve sparing surgery has been proposed to reduce the morbidity associated with radical surgery. When compared to the conventional surgery, nerve sparing surgeries are not only associated with better bowel and bladder function but also improvement in sexual dysfunction [32]. Studies compared the extent of surgery on sexual dysfunction. On comparing radical hysterectomy to more conservative treatment options like cervical conization, it was noted that women who had undergone radical hysterectomy reported worse sexual functions across all FSFI parameters [33]. Studies comparing radical hysterectomy with radical trachelectomy, revealed no difference in sexual health issues among women undergoing either of the procedure [34]. 
In cases of advanced cervical carcinoma multi modal therapy in the form of External beam radiotherapy (EBRT) and vaginal brachytherapy (VBT) with or without concurrent chemotherapy is utilised for treatment. Radiation is associated with an adverse impact on sexual health by causing vaginal stenosis and fibrosis, shortening of the vagina, atrophy of vaginal tissue, dyspareunia [35-39]. When radiation is combined with surgery, sexual dysfunction is worse as compared to those women who undergo treatment by surgery only $[28,40]$. In a retrospective study Frumovitz et al assessed sexual function among cervical cancer survivors with a long term follow up of seven years. They reported that radiotherapy had a negative impact on sexual function. Patients treated by radiotherapy reported a greater difficulty to be sexually aroused, decreased vaginal lubrication and decreased sexual satisfaction. Similar findings of statistically significant sexual dysfunction following treatment by radiation when compared to surgery were reported by Bermark et al and Jensen et al., [40-42] Radiation therapy also had adverse impact on bowel and bladder function. Women treated by radiation therapy reported incontinence and cystitis which affected self confidence and indirectly had a negative impact on the sexual health of women [43].

Women treated for cervical cancer experience sexual dysfunction after either surgery or radiotherapy. Complication secondary to vaginal morbidity and bowel and bladder dysfunction following surgery result in sexual health issues. These complications can be reduced with less radical, nerve sparing procedures. Radiation therapy used as either a primary form of treatment or adjuvant treatment after surgery, results in a higher degree of sexual dysfunction and morbidity [43].

\section{Assessment of Sexual Dysfunction}

In a survey of gynecologic oncologists conducted by Wiggins et al, it was found that less than half the clinicians obtained details regarding sexual history. Another obstacle in the management of sexual dysfunction is that of lack of proper methodology to screen and detect the problem. Hurdles in appropriate management of sexual dysfunctionare noted with both, the clinician as well as patients [44].

\section{Problems with Clinicians}

- Lack of knowledge and understanding of safe, viable options for treatment.

- Time consuming

- Need to establish baseline sexual function [45].

\section{Patient related Issues:[46]}

- Concerns of being dismissed

- Fear of physician being uncomfortable

- Feeling of lack of treatment options

- Cultural hindrance

- Ignorance

Various tools to assess sexual function: [49]

- The European Organization for Research and Treatment of Cancer Quality-of-Life Questionnaire Cervix Module 24
- Female Sexual Function Index (FSFI)- Most frequently utilised tool to assess sexual dysfunction and has good reliability

- Leiden Questionnaire

- Late Effects Normal Tissues (LENT)-Subjective, Objective, Management, Analytic (SOMA)- Jointly developed by EORTC and RTOG. It has both subjective and objective elements utilised to assess the impact of radiotherapy

Management of female sexual health issues in treated case of carcinoma breast and carcinoma cervix

Women treated for breast carcinoma and cervical carcinoma have various sexual health issue, needing a multidimensional approach to manage these issues. The treatment plan addresses the underlying issues which can be secondary to the disease pathology, treatment induced or psychological (anxiety and depression). Management of sexual health issue entails open communication, education and medical understanding [48].

\section{Female Sexual Interest/ Arousal Disorder}

Patient is reported to have reduced sexual interest if any 3 of the following are manifested [49]

- Decrease in interest in sexual activity

- Reduction in sexual thoughts

- Nonreceptive to a partner's initiation.

- Decreased sexual pleasure in $75-100 \%$ of her encounters

- Reduced arousal to any sexual cues

- Decreased genital/ non genital sensations

Sexual desire can be impacted adversely by various psychological factors like stress, negative body perception, trauma, depression and anxiety. Hence psychotherapy is successfully used to treat problems arising in terms of sexual desire. Cognitive behaviour therapy has been utilised for management of sexual dysfunction in cervical cancer survivors. Treatment was associated with improvement in desire, arousal, orgasm, satisfaction and overall improvement of FSFI score. It had no effect on pain, but did improve genital arousal and reduced distress $[50,51]$.

Flibanserin, a post synaptic serotonin receptor modulator was approved by US FDA for the management of hypoactive sexual desire disorder. Studies have revealed that treatment with flibanserin was associated with significant improvement in sexual desire and distress. Commonest side effects include dizziness, somnolence, nausea and fatigue. Flibanserin is contraindicated with the use of alcohol as it may cause severe hypotension and syncope [52, 53].

\section{Female orgasmic disorder}

Behavioural techniques involving graded stimulation of the genitals leads to increased arousal and orgasm. They have shown to be successful in almost 90 percent of the cases [54]. A combined approach involving both cognitive and behavioural interventions is recommended.

Medical management using various medications has been attempted, the drugs usually utilised are antidepressants. Among these paroxetine has the worst profile of adverse effects. Other 
SSRI including fluoxetine, sertraline or fluvoxamine may be used. Bupropion is another medication that can be considered for managing sexual dysfunction. In addition to antidepressant, one of the off label use of testosterone is utilising its libido enhancing effects. However in view of lack of studies, it is not recommended for use $[55,56]$.

\section{Dyspareunia/penetration disorder}

Treatment of gynaecologic malignancies is associated with dyspareunia. This may occur following treatment due to shortening, stenosis, decreased lubrication and atrophy of the vagina. Restoring vaginal lubrication, normalising the $\mathrm{pH}$, vaginal dilatation and pelvic floor therapy contribute to alleviate the symptoms.

Non hormonal treatment options include vaginal moisturizers and lubricants. These may be utilised several times to improve tissue quality, discomfort and to reduce dryness.

Various products are available which can be utilised as vaginal moisturisers [57].

\section{Polycarbophil based: [58-60]}

- They result in hydration of the underlying cells and cause maturation of vaginal epithelium.

- They results in reduction of bacterial vaginosis.

- Studies revealed improvement in dyspareunia and irritation comparable to the use of vaginal oestrogen. Vaginal oestrogen was superior to this compound for improvement in vaginal dryness.

\section{Hyaluronic acid based: [61, 62]}

- A glycosaminoglycan that leads to water retention and lubrication.

- It decreases vaginal $\mathrm{pH}$, causes a decrease in vaginal symptoms, atrophy and dryness.

\section{Hyaluronic acid, vitamin A, and vitamin E suppository: [63]}

- Effective in reducing vaginal dryness and dyspareunia.

\section{Vaginal Lubricants}

Lubricants result in temporary reduction in dryness, vaginal pain, mucosal tears and irritation. A large number of products are available over the counter. Water or silicone based products are preferred over petroleum based lubricants. Petroleum based lubricants are associated with unpleasant odour, vaginal infections. On the other hand water and silicone based lubricants not only have a better adverse effect profile, they are easily washed away by soap and water. For better efficacy lubricants must be applied by both partners [57].

\section{Low dose oestrogen therapy}

Low dose oestrogen therapy is efficacious in management of adverse genitourinary effects following menopause. Low dose oestrogen can be used in the form of cream, tablets and rings which improves symptoms due to vaginal atrophy without marked increase in serum oestrogen. However there are reports of in- creased risks of stroke, breast cancer and coronary heart disease as per the Women's Health Initiative report with the use of estrogen. Estrogen therapy is contraindicated in survivors of hormone dependant cancer. Hence initiation of therapy must be done after discussing the risk to benefit ratio. Estrogen be considered for women surviving non hormone receptor positive cancer in whom other options of treatment have failed [64-66].

\section{Non hormonal treatment}

Ospemifene is the only FDA approved non estrogenic medication for the management of moderate to severe dyspareunia due to vulvo-vaginal atrophy. It is a selective estrogen receptor modulator, which needs further evaluation for cancer survivors. Lignocaine has been used in breast cancer survivors for treatment of severe dyspareunia. When applied to vulvar vestibular tissue before penetration, it reduces distress and improves comfort. Further studies are needed to determine its application in gynaecologic cancers [67-69].

\section{Vaginal dilators}

Vaginal dilators are used in the prevention of vaginal stenosis secondary to pelvic radiotherapy. Although recommended, data supporting the use of dilators is conflicting and adherence is poor. They are beneficial in the management of dyspareunia and the vaginal morbidity secondary to the treatment and lack of estrogen $[57,70-72]$.

\section{Pelvic floor muscle exercise}

Strengthening pelvic floor musculature is an important aspect in sexual function. Patients who were managed with pelvic floor physical therapy have reported decrease in pain during intercourse, more pain free encounters and improvement in sexual function $[73,74]$.

\section{Conclusion}

Cancer survivors experience sexual dysfunction to a varying degree either secondary to the disease pathology or the treatment. Although an important aspect which impacts the quality of life of cancer survivors, it's a domain not adequately assessed or addressed. Furthermore, sexual dysfunction and poor quality of life can be easily addressed by simple measures.

Sexual health needs better understanding, addressal during medical evaluation after treatment completion. When needed, professionals specialising in this domain must be involved. In countries with low resource settings, management of sexual health issues is restricted by cultural and social factors. However these problems are frequent in cancer survivors and negatively impact the life of survivors. Hence its imperative to address this issue.

Further research and study is needed to understand the sexual health issues, identify risk factors, and determine treatment options.

\section{References}

[1]. Gabriel N. Hortobagyi, Jaime de la Garza Salazar, Kathleen Pritchard, Dino 
Amadori, Renate Haidinger, Clifford A. Hudis, et al. The global breast cancer burden: Variations in epidemiology and survival. Clin Breast Cancer. 2005 Dec; 6(5): 391-401. PMID: 16381622.

[2]. DeSantis CE, Lin CC, Mariotto AB, RebeccaL Siegel, Kevin D Stein, Joan L Kramer, et al. Cancer treatment and survivorship statistics, 2014. CA Cancer J Clin. 2014;64:252-71. PMID: 24890451.

[3]. Robert Michal Kowalczyk, Ida Cedrych, Zbigniew Lew-Starowicz, Krzysztof Nowosielski, Krzysztof Krzemieniecki, Marek Krzystanek, et al. Predictors of sexual function in women after treatment for breast cancer.Journal of Clinical Oncology. 2016; 34:15_suppl, 10071-10071.

[4]. Raggio GA, Butryn ML, Arigo D, Mikorski R, Palmer SC. Prevalence and correlates of sexual morbidity in long-term breast cancer survivors. Psychol Health. 2014;29(6):632-650. PMID: 24404999.

[5]. Susanna B Hummel, Jacques JDM van Lankveld, Hester SA Oldenburg, Daniela EE Hahn, Jacobien M Kieffer, Miranda A Gerritsma, et al. Efficacy of Internet-Based Cognitive Behavioral Therapy in Improving Sexual Functioning of Breast Cancer Survivors: Results of a Randomized Controlled Trial. Journal of Clinical Oncology. 2017; 35(12): 1328-1340. PMID: 28240966

[6]. Bray F, Ferlay J, Soerjomataram I, Siegel RL, Torre LA, Jemal A. Global cancer statistics 2018: GLOBOCAN of cancer incidence and mortality for 36 cancers in 185 countries. CA Cancer J Clin. 2018;68:394-424.

[7]. Shukla S, Bharti AC, Mahata S, Showket Hussain, Rakesh Kumar, Suresh Hedau, et al. Infection of human papillomaviruses in cancers of different human organ sites. Indian J Med Res. 2009;130:222-33. PMID: 19901431.

[8]. Basson R, Wierman ME, Van Lankveld J, Brotto L. Summary of the recommendations on sexual dysfunctions in women. The journal of sexual medicine. 2010 Jan 1;7(1):314-26.

[9]. Challenges reported by post-treatment cancer survivors in the livestrong surveys. Available online: http://www.livestrong.org/what-we-do/our-approach/reports-findings/survivor-survey-report/

[10]. Engel J, Kerr J, Schlesinger-Raab A,Hansjörg Sauer, Dieter Hölzel. Quality of life following breast-conserving therapy or mastectomy: results of a 5-year prospective study. Breast J 2004;10:223-31. PMID: 15125749.

[11]. Atisha D, Alderman AK, Lowery JC, Latoya E Kuhn, Jenny Davis, Edwin G Wilkins. Prospective analysis of long-term psychosocial outcomes in breast reconstruction: two-year postoperative results from the Michigan Breast Reconstruction Outcomes Study. Ann Surg. 2008;247:1019-28. PMID: 18520230 .

[12]. Ganz PA. Sexual functioning after breast cancer: a conceptual framework for future studies. Ann Oncol. 1997;8:105-7. PMID: 9093716.

[13]. Metcalfe KA, Semple J, Quan ML, Susan T Vadaparampil, Claire Holloway, Mitch Browne, et al. Changes in psychosocial functioning 1 year after mastectomy alone, delayed breast reconstruction, or immediate breast reconstruction. Ann Surg Oncol. 2012;19:233-41. PMID: 21674270.

[14]. Biglia N, Moggio G, Peano E, Paola Sgandurra, Riccardo Ponzone, Rossella E Nappi, et al. Effects of surgical and adjuvant therapies for breast cancer on sexuality, cognitive functions, and body weight. J Sex Med. 2010;7:1891900. PMID: 20233281.

[15]. Hidding JT, Beurskens CH, van der Wees PJ, Hanneke W M van Laarhoven, Maria W G Nijhuis-van der Sanden. Treatment related impairments in arm and shoulder in patients with breast cancer: a systematic review. PLoS One. 2014;9:e96748. PMID: 24816774.

[16]. Albornoz CR, Matros E, McCarthy CM, Anne Klassen, Stefan J Cano, Amy $\mathrm{K}$ Alderman, et al. Implant breast reconstruction and radiation: a multicenter analysis of long-term health-related quality of life and satisfaction. Ann Surg Oncol. 2014;21:2159-64. PMID: 24740825.

[17]. Ewertz M, Jensen AB. Late effects of breast cancer treatment and potentials for rehabilitation. Acta Oncol. 2011;50:187-93.

[18]. Safarinejad MR, Shafiei N, Safarinejad S. Quality of life and sexual functioning in young women with early-stage breast cancer 1 year after lumpectomy. Psychooncology. 2013;22:1242-8. PMID: 22777952.

[19]. Pagani O, Regan MM, Walley BA, Gini F Fleming, Marco Colleoni, István Láng, et al. Adjuvant exemestane with ovarian suppression in premenopausal breast cancer. N Engl J Med. 2014;371:107-18. PMID: 24881463.

[20]. Baumgart J, Nilsson K, Evers AS, Kallak TK, Poromaa IS. Sexual dysfunction in women on adjuvant endocrine therapy after breast cancer. Menopause. 2013 Feb 1;20(2):162-8

[21]. Halley MC, May SG, Rendle KA, Dominick L Frosch, Allison W Kurian. Beyond barriers: fundamental 'disconnects' underlying the treatment of breast cancer patients' sexual health. Cult Health Sex. 2014;16:1169-80. PMID: 25138386

[22]. Bhatla N0, Aoki D, Sharma DN, Sankaranarayanan R. Cancer of the cervix uteri. Int J Gynecol Obstet. 2018; 143: 22-36. PMID: 30306584.

[23]. Ries LA, Eisner MP, Kosary CL, Hankey BF, Miller BA, Clegg L, et al. SEER Cancer Statistics Review, 1975-2001. Bethesda, MD: National Cancer Institute; 2004.
[24]. Greenwald HP, McCorkle R. Sexuality and sexual function in long-term survivors of cervical cancer. J Womens Health (Larchmt). 2008;17(6):955-963. PMID: 18681816

[25]. Cleary V, Hegarty J. Understanding sexuality in women with gynaecological cancer. Eur J Oncol Nurs. 2011; 15(1): 38-45. PMID: 20584629.

[26]. Bartoces MG, Severson RK, Rusin BA, Schwartz KL, Ruterbusch JJ, Neale AV. Quality of life and self-esteem of long-term survivors of invasive and noninvasive cervical cancer. J Womens Health (Larchmt). 2009; 18: 655661. PMID: 19405862.

[27]. Maher EJ, Denton A. Survivorship, late effects and cancer of the cervix. Clin Oncol (R Coll Radiol). 2008;20(6): 479-487. PMID: 18515052.

[28]. E.R. Greimel, Raimund Winter, Karin S Kapp, Josef Haas. Quality of life and sexual functioning after cervical cancer treatment: a long-term follow-up study. Psychooncology.2009; 18(5): 476-482. PMID: 18702067.

[29]. Pernille T Jensen, Mogens Groenvold, Marianne C Klee, Ingrid Thranov, Morten Aa Petersen, David Machin. Early-stage cervical carcinoma, radical hysterectomy, and sexual function. A longitudinal study. Cancer. 2004; 100(1): 97-106. PMID: 14692029.

[30]. Quirine D Pieterse 1, Gemma G Kenter, Cornelis P Maas, Cor D de Kroon, Carien L Creutzberg, J Baptist M Z Trimbos, et al. Self-reported sexual, bowel and bladder function in cervical cancer patients following different treatment modalities: longitudinal prospective cohort study. Int. J. Gynecol. Cancer. 2013; 23(9): 1717-1725. PMID: 24172106.

[31]. Q D Pieterse, C P Maas, M M ter Kuile, M Lowik, M A van Eijkeren, JBMZ Trimbos, et al. An observational longitudinal study to evaluate miction, defecation, and sexual function after radical hysterectomy with pelvic lymphadenectomy for early-stage cervical cancer, Int. J. Gynecol. Cancer. 2006; 16(3): 1119-1129. PMID: 16803495.

[32]. Marcello Ceccaroni 1, Giovanni Roviglione, Emanuela Spagnolo, Paolo Casadio, Roberto Clarizia, Michele Peiretti, et al. Pelvic dysfunctions and quality of life after nerve-sparing radical hysterectomy: A multicenter comparative study. Anticancer Res. 2012; 32(2): 581-588. PMID: 22287748.

[33]. Taejong Song, Chel Hun Choi, Yoo-Young Lee, Tae-Joong Kim, Jeong-Won Lee, Byoung-Gie Kim, et al. Sexual function after surgery for early-stage cervical cancer: is there a difference in it according to the extent of surgical radicality?. J. Sex. Med. 2012; 9(6): 1697-1704. PMID: 22548752.

[34]. Ligita Paskeviciute Froeding 1, Christian Ottosen, Helle Rung-Hansen, Danny Svane, Berit Jul Mosgaard, Pernille Tine Jensen. Sexual functioning and vaginal changes after radical vaginal trachelectomy in early stage cervical cancer patients: a longitudinal study. J. Sex. Med. 2014; 11(2): 595-604. PMID: 24286464

[35]. A Katz 1, E Njuguna, E Rakowsky, A Sulkes, J Sulkes, E Fenig. Early development of vaginal shortening during radiation therapy for endometrial or cervical cancer. Int. J. Gynecol. Cancer.2001; 11: 234-235. PMID: 11437931.

[36]. LR Schover, M Fife, DM Gershenson. Sexual dysfunction and treatment for early stage cervical cancer. Cancer.1989; 63(1): 204-212. PMID: 2910419.

[37]. K Bergmark, E Avall-Lundqvist, PW Dickman, L Henningsohn, G Steineck. Vaginal changes and sexuality in women with a history of cervical cancer. N. Engl. J. Med. 1999; 340(18): 1383-1389. PMID: 10228188.

[38]. AH Brand, CA Bull, B Cakir. Vaginal stenosis in patients treated with radiotherapy for carcinoma of the cervix. Int. J. Gynecol. Cancer.2006; 16(1): 288-293. PMID: 16445647.

[39]. D W Bruner, R Lanciano, M Keegan, B Corn, E Martin, G E Hanks. Vaginal stenosis and sexual function following intracavitary radiation for the treatment of cervical and endometrial cancer. Int. J. Radiat. Oncol. Biol. Phys. 1993; 27(4): 825-830. PMID: 8244811.

[40]. Michael Frumovitz, Charlotte C Sun, Leslie R Schover, Mark F Munsell, Anuja Jhingran, J Taylor Wharton, et al. Quality of life and sexual functioning in cervical cancer survivors. J. Clin. Oncol.2005; 23(30): 7428-7436. PMID: 16234510

[41]. K Bergmark, E Avall-Lundqvist, PW Dickman, L Henningsohn, G Steineck. Vaginal changes and sexuality in women with a history of cervical cancer. $N$ Engl J Med. 1999;340(18):1383-1389. PMID: 10228188

[42]. Pernille T Jensen, Mogens Groenvold, Marianne C Klee, Ingrid Thranov, Morten Aa Petersen, David Machin. Early-stage cervical carcinoma, radical hysterectomy, and sexual function. A longitudinal study. Cancer. 2004;100(1):97-106. PMID: 14692029.

[43]. Laura B Huffman, Ellen M Hartenbach, Jeanne Carter, Joanne K Rash, David $M$ Kushner. Maintaining sexual health throughout gynecologic cancer survivorship: A comprehensive review and clinical guide. Gynecol Oncol. 2016; 140(2): 359-368. PMID: 26556768.

[44]. Wiggins DL, Wood R, Granai CO, Don S Dizon. Sex, intimacy, and the gynecologic oncologists:survey results of the new England association of gynecologic oncologists (NEAGO). J Psychosoc Oncol. 2007;25(4):61-70. PMID: 18032265.

[45]. Quirk F, Haughie S, Symonds T. The use of the sexual function question- 
naire as a screening tool for women with sexual dysfunction. J Sex Med. 2005;2(4):469-77. PMID: 16422841.

[46]. Marwick C. Survey says patients expect little physician help on sex. JAMA. 1999;281: 2173-4. PMID: 10376552.

[47]. Abhishek Shankar, Neelam Prasad, Shubham Roy, Abhijit Chakraborty, Aalekhya Sharma Biswas, Jaineet Patil, et al. Sexual Dysfunction in Females after Cancer Treatment: An Unresolved Issue. Asian Pac J Cancer Prev. 2017;18(5):1177-1182. PMID: 28610399.

[48]. Krychman ML, Pereira L, Carter J, Alison Amsterdam. Sexual oncology:sexual health issues in women with cancer. Oncology. 2006;71: 18-25. PMID: 17347586.

[49]. American Psychiatric Association. Diagnostic and statistical manual of mental disorders (DSM-5 ${ }^{\oplus}$. American Psychiatric Pub; 2013 May 22.

[50]. Lori A Brotto, Johannes Bitzer, Ellen Laan, Sandra Leiblum, Mijal Luria. Women's sexual desire and arousal disorders. J Sex Med.2010; 7(1 Pt 2): 586-614. PMID: 20092454.

[51]. Lori A Brotto, Yvonne Erskine, Mark Carey, Tom Ehlen, Sarah Finlayson, Mark Heywood, et al. A brief mindfulness-based cognitive behavioral intervention improves sexual functioning versus wait-list control in women treated for gynecologic cancer. Gynecol. Oncol. 2012; 125(2): 320-325. PMID: 22293042.

[52]. FDA Joint Meeting of Bone, Reproductive and Urologic Drugs Advisory Committee and the Drug Safety and Risk Management Advisory Committee, Flibanserin for the treatment of hypoactive sexual desire disorder in premenopausal women, NDA 022526. Advisory Committee Briefing Document, 2015.

[53]. James A Simon, Sheryl A Kingsberg, Brad Shumel, Vladimir Hanes, Miguel Garcia, Michael Sand.Efficacy and safety of flibanserin in postmenopausal women with hypoactive sexual desire disorder: results of the SNOWDROP trial. Menopause. 2014; 21(6): 633-640. PMID: 24281236.

[54]. Ellen Laan, Alessandra H Rellini, Tricia Barnes.Standard operating procedures for female orgasmic disorder: consensus of the international society for sexual medicine. J. Sex. Med.2013; 10(1): 74-82. PMID: 22970851.

[55]. Debra L Barton, Donald B Wender, Jeff A Sloan, Robert J Dalton, Ernie P Balcueva, Pamela J Atherton, et al. Randomized controlled trial to evaluate transdermal testosterone in female cancer survivors with decreased libido; North central cancer treatment group protocol N02C3. J Natl Cancer Inst. 2007;99: 672-9. PMID: 17470735.

[56]. H Croft, E Settle, T Houser, S R Batey, R M Donahue, J A Ascher. A placebo-controlled comparison of the antidepressant efficacy and effects on sexual functioning of sustained-release bupropion and sertraline. Clin Ther. 1999;21:643-58. PMID: 10363731.

[57]. J. Carter, D. Goldfrank, LR Schover. Simple strategies for vaginal health promotion in cancer survivors. J. Sex. Med. 2011; 8(2): 549-559. PMID: 20722792.

[58]. JAWM van der Laak, LMT de Bie, H de Leeuw, PCM de Wilde, AGJM Hanselaar. The effect of Replens on vaginal cytology in the treatment of postmenopausal atrophy: cytomorphology versus computerised cytometry. J Clin. Pathol.2002; 55: 446-451. PMID: 12037029.

[59]. Wu JP, Fielding SL, Fiscella K. The effect of polycarbophil gel (Replens ${ }^{\mathrm{TM}}$ ) on bacterial vaginosis: A pilot study. European Journal of Obstetrics \& Gynecology and Reproductive Biology. 2007 Jan 1;130(1):132-6.

[60]. M Bygdeman, M Swahn. Replens versus dienoestrol cream in the symptomatic treatment of vaginal atrophy in postmenopausal women. Maturitas.1996; 23(3): 259-263. PMID: 8794418
[61]. Junya Chen, Li Geng, Xuehong Song, Hongxia Li, Nicola Giordan, Qinping Liao. Evaluation of the efficacy and safety of hyaluronic acid vaginal gel to ease vaginal dryness: a multicenter, randomized, controlled, open-label, parallel- group, clinical trial. J. Sex. Med.2013; 10(6): 1575-1584. PMID: 23574713.

[62]. Murat Ekin, Levent Yaşar, Kadir Savan, Muzaffer Temur, Mehmet Uhri, Işıl Gencer, et al. The comparison of hyaluronic acid vaginal tablets with estradiol vaginal tablets in the treatment of atrophic vaginitis: a randomized controlled trial. Arch. Gynecol. Obstet.2011; 283(3): 539-543. PMID: 20135132.

[63]. D Constantino, C Guaraldi. Effectivness and safety of vaginal suppositories for the treatment of vaginal atrophy in posmenopausal women: an open, non-controlled clinical trial. Eur. Rev. Med. Pharmacol. Sci.2008; 12(6): 411-416. PMID: 19146203.

[64]. David D Rahn, Cassandra Carberry, Tatiana V Sanses, Mamta M Mamik, Renée M Ward, Kate V Meriwether, et al. Vaginal estrogen for genitourinary syndrome of menopause: a systematic review. Obstet. Gynecol. 2014; 124(6): 1147-1156. PMID: 25415166.

[65]. JA Suckling, A Lethaby, R Kennedy.Local estrogen for vaginal atrophy in postmenopausal women. Cochrane Database Syst. Rev. 2006; 4: CD001500. PMID: 14583935.

[66]. Jacques E Rossouw, Garnet L Anderson, Ross L Prentice, Andrea Z LaCroix, Charles Kooperberg, Marcia L Stefanick, et al. Risks and benefits of estrogen plus progestin in healthy post- menopausal women: Principal results from the women's health initiative randomized controlled trial. J. Am. Med. Assoc. 2002; 288(3): 321-333. PMID: 12117397.

[67]. D Portman, S Palacios, RE Nappi, AO Mueck. Ospemifene, a non-oestrogen selective oestrogen receptor modulator for the treatment of vaginal dryness associated with postmenopausal vulvar and vaginal atrophy: a randomised, placebo-controlled, phase III trial. Maturitas.2014; 78(2): 91-98. PMID: 24679891.

[68]. GA Bachmann, JO Komi. Ospemifene effectively treats vulvovaginal atrophy in postmenopausal women: results from a pivotal phase 3 study. Menopause. 2010; 17(3): 480-486. PMID: 20032798.

[69]. MF Goetsch, JY Lim, AB Caughey. A Practical Solution for Dyspareunia in Breast Cancer Survivors: A Randomized Controlled Trial. J. Clin. Oncol. 2015; 33(30): 3394-400. PMID: 26215946.

[70]. TPMiles, N Johnson. Vaginal dilator therapy for women receiving pelvic radiotherapy.Cochrane Database Syst. Rev. 2010; 9: CD007291. PMID: 20824858.

[71]. N. Johnson, TP. Miles, P. Cornes. Dilating the vagina to prevent damage from radiotherapy: systematic review of the literature. BJOG.2010; 117(5): 522-531. PMID: 20163407.

[72]. Lois C Friedman, Rita Abdallah, Mark Schluchter, Ashok Panneerselvam, Charles A Kunos. Adherence to vaginal dilation following high dose rate brachytherapy for endometrial cancer. Int. J. Radiat. Oncol. Biol. Phys. 2011; 80(3): 751-757. PMID: 20619551.

[73]. Lior Lowenstein, Ilan Gruenwald, Irena Gartman, Yoram Vardi. Can stronger pelvic muscle floor improve sexual function?. Int. Urogynecol. J.2010; 21(5): 553-556. PMID: 20087572.

[74]. Corrie Goldfinger, Caroline F Pukall, Evelyne Gentilcore-Saulnier, Linda McLean, Susan Chamberlain. A prospective study of pelvic floor physical therapy: pain and psychosexual outcomes in provoked vestibulodynia. J. Sex. Med.2009; 6(7): 1955-1968. PMID: 19453890. 